\title{
Transmission Line Maintenance Scheduling Based on Graph Coloring
}

\author{
Yu Hongtao* \\ Institute of Automation \\ Shenyang Institute of Engineering \\ Shenyang, China \\ neu970773@sohu.com \\ * Corresponding Author \\ Ma Yang \\ Institute of Automation \\ Shenyang Institute of Engineering \\ Shenyang, China \\ mayanghc@163.com
}

\author{
Han Xichang \\ Institute of Automation \\ Shenyang Institute of Engineering \\ Shenyang, China \\ Hanxc@sie.edu.cn
}

\begin{abstract}
In order to improve the efficiency of transmission line maintenance scheduling, a multi-objective and multiscale mathematical model is proposed. The coloring problem of graph theory and analytic hierarchy process are combined, and various constrains are considered in the model. In order to deal with discrete variables problems, a new method based on harmony search algorithm combined with ant colony algorithm is proposed. The harmony search algorithm is developed based on an analogy with music improvisation process. Musical performers seek to find pleasing harmony as determined by aesthetic standard, just as the optimization process seeks to find global optimization solution. Harmony search algorithm is suitable for solving the problem of continuous variable problem, so it is improved in this paper. The number of color got by using ant colony algorithm is the first stage, and the coloring order obtained by using improved harmony search algorithm is the second stage. Furthermore we discuss the application of our models to a case, and simulation results show that the proposed model and algorithm are effective.
\end{abstract}

Keywords-transmission line; maintenance scheduling; graph coloring; harmony search algorithm; multi-objective optimization

\section{INTRODUCTION}

With the rapid development of industry and science and technology, the structure and scale of the transmission line become more and more complex. Increasing the size of the grid increase the transmission capacity, factors that affect the reliable operation of the power system also increase. Regular maintenance of the transmission lines is to ensure safe and reliable operation of power system. Maintenance activities are often under conditions of transmission line outage, line outage may cause the system to reduce the power supply reliability and transmission capacity. At present, most of the regional transmission line maintenance planners rely on the original manual method or simple computer program, which is difficult to make a reasonable maintenance plan. Reasonable arrangement for transmission line maintenance has become an important subject line administration.

Maintenance scheduling has become a hot research. Yare[1,2] applid respectively discrete multi PSO and PSO to make generator maintenance plan. Geetha[3] studied coordinated preventive maintenance planning problem under competitive mechanism. None of the above document solved transmission line maintenance. Jiang and Zhao[4] used traditional methods to make transmission line maintenance plan, which need to spend a lot of time for large-scale maintenance tasks. Gao[5], and Liu[6,7] proposed optimization model based on characteristic of line maintenance, and the application of intelligent algorithm was carried out for solving the model. Unfortunately, they did not consider multiple sets of maintenance. Graph coloring will be made use of in this paper to build transmission line maintenance model. What's more, line maintenance plan is NP-hard problem, which is difficult to solve. This paper presents an improved harmony search algorithm to solve the problem.

\section{PROBLEM FORMULATION}

Firstly, each bureau provides maintenance tasks when staff scheduling makes the transmission line maintenance plan. Secondly, staff scheduling analyzes the maintenance tasks. Finally, staff scheduling makes a reasonable maintenance plan based on maintenance tasks characteristic. The parameters and decision factors of the model are as follows.

a) The same power administration can not repair two parallel lines at the same time

b) The same substation can not be two parallel lines at the same time

c) The urgent degree of the mission 


\section{THE MODEL FOR TRANSMISSION LINE MAINTENANCE SCHEDULING}

When transmission line maintenance plan is made, the parallel lines can't overhaul in the same time. Correspondingly, when color a graph, the adjacent vertex can't color the same color. So the parallel lines can be taken regard as the adjacent vertex of coloring problem. As a result, the problem of electric power line overhaul plan be been described by coloring problem.

Suppose the transmission line maintenance task is $A=\left\{a_{1}, a_{2}, \cdots, a_{n}\right\}, A$ is looked on as the set of the vertex in coloring problem. If two lines are parallel, the two lines are called conflicting lines. The conflicting lines are represented by disorder couple gather $E, E$ is taken regard as the set of the edge in coloring problem, if $a_{i}$ and $a_{j}$ conflict each other, then $\left(a_{i}, a_{j}\right) \in E$. Suppose time quantum is $T=\left\{t_{1}, t_{2}, \cdots, t_{i} \cdots\right\}, T$ represents the number of time quantum when overhaul lines satisfy the requests that the conflicting lines can't overhaul in the same time. Therefore, the undirected graph $G=(A, E)$ can show the problem of electric power line overhaul, and the aim $T$ is that the minimum amount of color is obtained by coloring.

Three objective functions are considered are considered in this paper, which can be stated as follows.

The first objective function is the minimization of the time quantum. The total overhaul time is certain for ransmission line maintenance, so the minimum time quantum can make each maintenance task have enough overhaul time. The time quantum can be got based on the model of vertex coloring, which is discribed as follows:

$$
f_{1}^{*}=\min f_{1}=\min t_{\max }
$$

Where $t_{\max }$ is the minimum color of vertex coloring, that is to say, $t_{\max }$ is the time quantum of ransmission line maintenance.

The second objective function is the economic lost. A certain overhaul time will be expected for some maintenance tasks. If the actual time is later than the expected time, it will result in economic lost at a certain degree. The second objective function can be stated as follows:

$$
f_{2}^{*}=\min f_{2}=\min k \sum_{i=1}^{n} \max \left(\eta_{\text {expect }}-\eta_{\text {fact }}, 0\right)
$$

Where $\eta_{\text {expect }}=\left\{\eta_{1}, \eta_{2}, \cdots, \eta_{n}\right\}$ is the expected overhaul time, $\eta_{\text {fact }}=\left\{\eta_{1}, \eta_{2}, \cdots, \eta_{n}\right\}$ is the actual overhaul time, $k$ is a parameter of economic lost that can be got by historical data

The third objective function is the average degree of maintenance tasks. There is too much or too little maintenance tasks at the same time quantum, which is not expected for maintenance staff, so the work balance degree is consided, which can been defined as:

$$
f_{3}^{*}=\min f_{3}=\min \frac{1}{t_{\max }-1} \sum_{i=1}^{t_{\max }}\left(s_{i}-\bar{s}\right)^{2}
$$

where $s_{i}$ is the number of jobs in each time quantum.

Because the three objectives are conflicting, a new objective is defined:

$$
f=\min \sum_{i=1}^{3} \omega_{i} \square f_{i}
$$

where $\omega_{i}$ is got according to analytic hierarchy process.

$$
A=\left(\begin{array}{lll}
1 & \frac{1}{4} & 1 \\
4 & 1 & 3 \\
1 & \frac{1}{3} & 1
\end{array}\right)
$$

$A$ is the judgment matrix that is given by expert in electric line overhaul. Then the $\omega_{i}$ is got, which is $\omega_{1}=0.174 \quad, \quad \omega_{2}=0.634 \quad, \quad \omega_{3}=0.192 \quad, \quad$ and $C I=0.0048, C R=0.0083<0.10$. So matrix $A$ has satisfactory consistency.

\section{THE SOLUTION ALGORITHM}

Ant colony algorithm(ACO)[8] is proposed by Dorigo $\mathrm{M}$ and some other Italian scholars, which simulates the ant finding path behavior in nature. The ant colony algorithm solves the question according to the method of ant finding food and goes back to the nest. Because ACO is suitable for solving the problem of discrete variable, the number of color for graph coloring is got by it.

The harmony search (HS) algorithm[9,10] is originally developed by Geem et al, which is used mainly to solve continuous variables problem. It is difficult to deal with integer programming problem. What's more, it is easy to fall into local optimization, so an improved HS(IHS) is proposed to optimize maintenance constraints in this paper.

The IHS in this paper can be described as follow, and the procedure is given in Fig. 1.

Step 1: initialize the problem and algorithm parameters

In this step, the optimization problem is specified as follows:

$$
\text { Minimize } f(x)
$$

$$
\text { Subject to } x_{i} \in X_{i}, i=1,2, \ldots, N
$$

where $f(x)$ is an objective function; $\mathrm{x}$ is a solution vector composed of decision variable $x_{i} ; \mathrm{N}$ is the number of decision variables; $X_{i}$ is the set of the possible range of values for each decision variable, that is, $X_{i}=\left\{x_{i}(1), x_{i}(2), \ldots, x_{i}(K)\right\}$; and $\mathrm{K}$ is the number of possible values for the decision variables. In addition, the harmony memory size (HMS), harmony memory considering rate (HMCR), pitch adjusting rate (PAR) and the number of improvisations (NI) are given in this step. 
For distribution network reconfiguration problem, the objective function is loss minimization, switches that can be opened or closed is the decision variable; the number of decision variables $\mathrm{N}$ is the number of branches in the network.

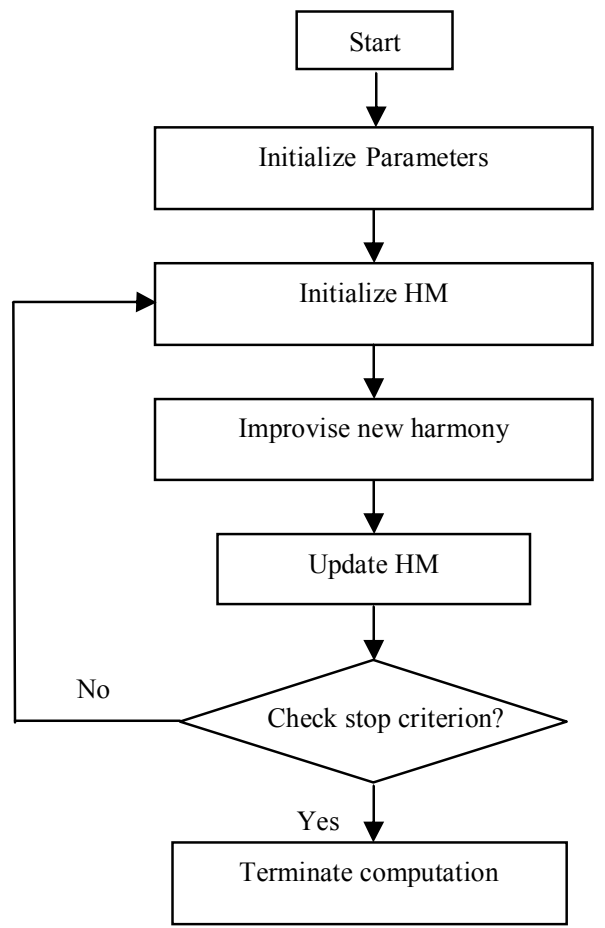

Figure 1. Optimization procedure of harmony search algorithm

Step 2: initialize the harmony memory

In this step, the harmony memory(HM) matrix is filled with as many randomly generated solution vectors as the HMS.

For transmission line maintenance scheduling problem, $x_{i}$ of HM is order variable. The same number represents that transmission line is overhauled at the same time quantum. If solution vector is not infeasible, the number of $x_{i}$ can be changed.

Step 3: improvise a new harmony

In this step, a new harmony is improvised, which bases on three rules:

a) random selection

In the random selection process, the new harmony improvisation process bases on random operation, that is to say, the new harmony vector is generated randomly.

b) memory consideration

In the memory consideration process, a harmony vector in HMS is selected by roulette methods, and then the new harmony vector bases on cross operation for the selected vector.

c) pitch adjustment

In the pitch adjustment process, a harmony vector in HMS is selected by roulette methods, and then the new harmony vector bases on insert operation for the selected vector.

Step 4: update the harmony memory

The new harmony vector replaces the worst harmony in the HM, if its objective function value is better than that of the worst harmony.
Step 5: check stopping criterion

Steps 3 and 4 are repeated until the termination criterion (NI) is satisfied.

\section{EXPERIMENTAL RESULTS}

In this section, we present some numerical examples to consider the model behavior and the near-optimal solutions. Parameters of algorithm is as in [8] and [9]. The maintenance tasks are shown in TABLE I, and maintenance constraints are given in TABLE II .

\begin{tabular}{|c|c|c|c|}
\hline Maintenance line & Start time & End time & Urgent degree \\
\hline 1 & 1 & 5 & 5.4 \\
\hline 2 & & & 5.4 \\
\hline 3 & & & 3.7 \\
\hline 4 & & & 4.1 \\
\hline 5 & 7 & 12 & 0.2 \\
\hline 6 & & & 0.7 \\
\hline 7 & & & 2.5 \\
\hline 8 & & & 2.2 \\
\hline 9 & & & 1.8 \\
\hline 10 & & & 0.4 \\
\hline 11 & & & 1.3 \\
\hline 12 & & & 1.8 \\
\hline 13 & & & 2.4 \\
\hline 14 & & & 2.5 \\
\hline 15 & & & 4.4 \\
\hline 16 & & & 4.1 \\
\hline 17 & & & 4.5 \\
\hline 18 & & & 5.8 \\
\hline 19 & & & 6.2 \\
\hline 20 & & & 8.2 \\
\hline 21 & & & 9.1 \\
\hline 22 & & & 8.3 \\
\hline 23 & & & 7.1 \\
\hline 24 & & & 6.4 \\
\hline 25 & & & 6.8 \\
\hline 26 & & & 8.3 \\
\hline 27 & & & 8.7 \\
\hline 28 & & & 7.4 \\
\hline 29 & & & 7.1 \\
\hline 30 & 6 & 10 & 5.8 \\
\hline
\end{tabular}


TABLE II . RESTRICTIONS OF MAINTENANCE RELATIONSHIP

\begin{tabular}{cc}
\hline Maintenance line & Same time quantum overhaul \\
\hline 2,10 & no \\
6,18 & no \\
9,19 & no \\
20,22 & no \\
\hline
\end{tabular}

TABLE III. compares the proposed algorithm with the results of ACO, PSO and GA algorithm. According to TABLE III., we have found that the different results are obtained by different algorithms. The optimal result is got by the proposed algorithm.

TABLE III.. EXPERIMENT RESULTS FOR 30 SETS OF DATA

\begin{tabular}{ccccc}
\hline Number of colors & Algorithm & Mean & Best & Worst \\
\hline & ACO & 91.5339 & 89.5461 & 92.9054 \\
2 & PSO & 91.8231 & 90.0125 & 93.2145 \\
& GA & 91.6120 & 89.7125 & 92.9521 \\
& IHS & 90.5382 & 89.3703 & 91.4542 \\
\hline
\end{tabular}

TABLE IV. shows the optimal maintenance strategy. The set $\{27,1,22,26,16,30,10,6,5,12,11,9,13,4,7\}$ and $\{2,28,29,19,25,17,21,3,8,15,20,18,23,24$, 14 \} can overhaul at the same time. What' more, if the number of tasks is too big to overhaul simultaneously, the maintenance order can be got by TABLE IV . too . Fig. 2 shows the stem graph of maintenance order.

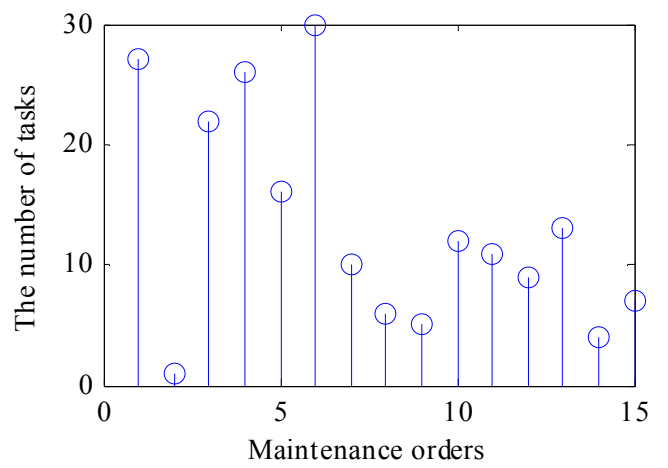

(a)

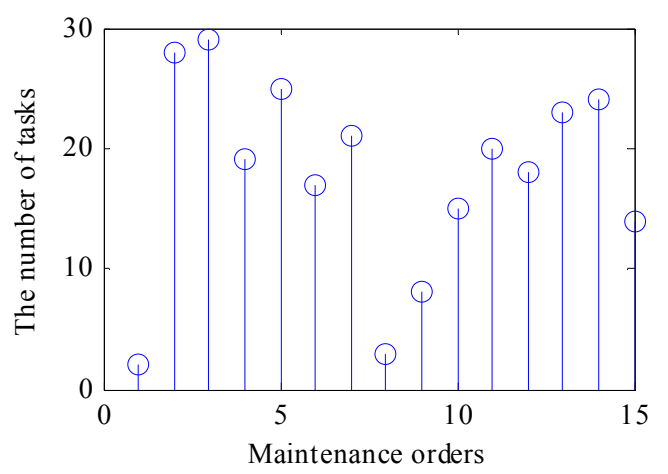

(b)

Figure 2. Maintenance orders for two sets
TABLE IV. MAINTENANCE ORDERS FOR 30 SETS OF DATA

\begin{tabular}{cc}
\hline Number of colors & Optimal maintenance order \\
\hline 2 & $27,1,22,26,16,30,10,6,5,12,11,9,13,4,7$ \\
& $2,28,29,19,25,17,21,3,8,15,20,18,23,24,14$ \\
\hline
\end{tabular}

When we use this model to make transmission line maintenance schedule, not only the time quantum but also the overhaul order of each maintenance task are can been got according to the results, that is to say, which job is in which time quantum is can been got by the simulation results.

\section{CONCLUSIONS}

A multi-objective and multi-scale mode is presented based on the analysis of transmission line maintenance scheduling problem, and various constrains are taken into account. The coloring problem of graph theory and analytic hierarchy process are combined to make scheduling by using ACO and IHS. Simulation results show that we can make transmission line maintenance plan based on the proposed model quickly, and the IHS is superior to ACO, PSO and GA in quality.

\section{ACKNOWLEDGMENT}

This work was supported in part by the National Natural Science Foundation of China under Grant 61372195, 61304069 and 61371200, Liaoning Province Dr. started fund under Grant 201501087 and Science Research General Project of Liaoning Education Department under Grant L2014530, L2014517, L2015367 and L2015366.

\section{REFERENCES}

[1] Yare Y, Venayagamoorthy G K. Optimal maintenance scheduling of generators using multiple swarms-MDPSO framework [J], Engineering Applications of Artificial Intelligence, 2010, 23(6) : 895-910.

[2] Yare Y, Venayagamoorthy G K, Aliyu U O. Optimal generator maintenance scheduling using a modified discrete PSO [J], Generation, Transmission \& Distribution, 2008, 2(6): 834-846.

[3] Geetha T, Shanti Swarup K. Coordinated preventive maintenance scheduling of GENCO and TRANSCO in restructured power systems [J], International Journal of Electrical Power \& Energy Systems, 2009, 31(10): 626-638.

[4] Zhao D F, Duan X F, Zhang Ll. Maintenace scheduling of power transmission equipment considering equipment condition and system risk[J], Journal of Xi'an Jiaotong University, 2012, 46(3): 6-11.

[5] Gao W H, Wang J X, Lu J M, Xu K Q. Maintenance schedule of transmission system based on equal risk[J], Automation of Electric Power Systems, 2012, 36(7): 6-11

[6] Liu W Y, Xie C, Wen J, Wang J M. Optimization of transmission network maintenace scheduling based on niche multi-objective particle swarm algortithm[J], Proceeding of the CSEE, 2013, 33(4): 141-146.

[7] Liu W Y, Xie C, Wen J, Wang J M, Li J. Multi-objiective decision making method for maintenance scheduling of transmission network[J], Power System Technology, 2013, 37(2): 471-476. 
[8] Zhao H P, Hu K, Li J W. Evolutionary stable strategy ant colony algorithm for solvign graph coloring[ J], 2013, 30(5): 22-24.40.

[9] Geem Z W, Kim J H, Loganathan G V. A new heuristic optimization algorithm: harmony search[J]. Simulation, 2001, 76(2): 60-68
[10] Zou D X, Gao L Q, Li S, Wu J H. Solving 0-1 knapsack problem by a novel global harmony search algorithm [J], Applied Soft Computing, 2011, 11(2): 1556-1564. 